\title{
Prevalence of Nocturnal Enuresis among Saudi Children Population
}

Elham H. Alhifthy ${ }^{1}$, Lura Habib ${ }^{2}$, Azahir Abu Al-Makarem ${ }^{3}$, Maryam AlGhamdi ${ }^{4}$, Doaa Alsultan ${ }^{5}$, Fatemah Aldhamer ${ }^{6}$, Rawan Buhlagah ${ }^{5}$, Fatimah M. Almubarak ${ }^{5}$, Eatesam Almufadhi ${ }^{7}$, Ghaida M. Bukhamsin ${ }^{5}$, Maria H. Zadah ${ }^{8}$

1. Clinical Sciences, Pediatrics, Developmental and Behavioural Pediatrics, Princess Nourah Bint Abdulrahman University, College of Medicine, Riyadh, SAU 2. Medicine, Taif University, Taif, SAU 3. Medicine, Umm Alqura University (UQU), Makkah, SAU 4. Medicine, Aljouf University, Al-Jouf, SAU 5. Medicine, King Faisal University, Riyadh, SAU 6. Medicine, Princess Nourah University, Riyadh, SAU 7. Medicine, Al Jouf University, Al-Jouf, SAU 8. Medicine, Princess Nourah Bint Abdulrahman University, Riyadh, SAU

Corresponding author: Elham H. Alhifthy, ehalhifthy@pnu.edu.sa

\section{Abstract}

\section{Background}

Nocturnal enuresis (NE) is the involuntary urination that occurs while asleep after an age when bladder control at night is expected. It has a global incidence of $1.4 \%-28 \%$ among 6-12 years old children. The aim of this study is to show the prevalence, risk factors, types of provided treatment of enuresis among studied children in Kingdom of Saudi Arabia (KSA).

\section{Methods}

A cross-sectional descriptive study was carried out among Saudi children, 3-12 years of age, from different cities in Saudi Arabia, during the period from 20 October to 20 November 2019. Data was collected by using a pre-designed questionnaire that was distributed online and included questions designed to fulfill the study objectives.

\section{Results}

This study reported that $31.2 \%$ of Saudi children of the chosen ages are suffered from enuresis, the majority occurred at day and night by $55.1 \%$ while $43.9 \%$ occurred only at night. Participants described types of provided treatment as follows: behavioral modification was the most commonly used by $31.6 \%$ followed by pharmacological intervention (29.6\%), bed-wetting alarm (6.8\%), exercises to strengthen the bladder muscles $(6.2 \%)$ and surgical intervention reported by $1.5 \%$ only. It was found that the improvement of enuresis on treatment occurred in $43.6 \%$ of cases. There was a significant reduction of the prevalence of NE with age (peak is $63.6 \%$ in 5-7 years old) but no significant correlation was found with gender $(\mathrm{p}=0.104)$. However, there was a significant correlation with parent having history of $\mathrm{NE}(p=0.001)$.

Received 12/11/2019

Review began 12/18/2019 Review ended 01/07/2020 Published 01/15/2020

() Copyright 2020

Alhifthy et al. This is an open access article distributed under the terms of the Creative Commons Attribution License CC-BY 3.0., which permits unrestricted use, distribution, and reproduction in any medium, provided the original author and source are credited.

\section{Conclusion}

The study reported that $31.2 \%$ of children found to have nocturnal enuresis; $43.9 \%$ of those had nocturnal enuresis alone. There were no significant correlations between nocturnal enuresis and child gender while it significantly correlated with child's age and having a family history of NE. Behavioral modification therapy was the most commonly provided treatment followed by pharmacological intervention; improvement occurred in less than half of the cases with treatment.

Categories: Pediatrics, Psychiatry, Psychology

Keywords: nocturnal enuresis, risk factors, provided treatment

\section{Introduction}

Nocturnal enuresis (NE) is the involuntary urination that occurs while asleep after an age when bladder control at night is expected (Involuntary urination that happens during the day is known as diurnal enuresis.) [1]. Nocturnal enuresis is a common problem in children and teenagers. NE is the second most common disorder affecting children 6-14 years of age after allergic disorders [2]. The global incidence of enuresis in children 6-12 years of age was shown to be $15 \%-25 \%$ in one study [3]. While a study in Egypt showed an incidence of $18 \%$ among children $9 \pm 2$ years old [ 4 ].

Enuresis may be classified into primary and secondary forms. Primary enuresis is when a child $>5$ years of age has never achieved a period of complete dryness for six or more months in a row [5]. While secondary enuresis is a condition that develops at least six months or several years after a child has achieved a period of complete dryness. 
There are a variety of proposed causes of NE including: low bladder capacity, insufficient antidiuretic hormone production at night, familial/genetic causes, upper airway obstructions or less often structural problems in the urinary tract or nervous system [6]. Other causes include a variety of development, diabetes insipidus, urinary tract infections, stressful circumstances in school or family [7].

Nocturnal enuresis may lead to low self-esteem, a sense of failure, chronic stress and it may affect the child's social life. It can trigger a range of behavioral, psychological and social problems. Therefore, it is important to identify children at risk and perform therapeutic measures [8].

Nocturnal enuresis is known to have multiple comorbidities which raises the importance of recognizing it and looking for associated symptomatology. Those comorbidities include neuropsychiatric problems like intellectual disability, attention deficit hyperactivity disorder (ADHD), psychological disorders and low selfesteem. Urinary tract infections, obstructive sleep apnea, diabetes, and low hormones as ADH are other disorders known to be associated with NE [3,9].

This study aims to find the prevalence of nocturnal enuresis within children in Saudi Arabia, its risk factors and provided management modalities of enuresis among 3-12-year-old children in different cities in KSA.

\section{Materials And Methods \\ Participants and methods}

A cross-sectional descriptive study was carried out among 2148 Saudi children aged 3-12 years from different Saudi provinces, which represent different localities and cultures of the kingdom. It was conducted during the period from 20 October to 20 November 2019. The sample size of 2148 was calculated according to the sample size using the equation of $n=z^{2} p(1-p) / e^{2}(n=$ sample size, $z=$ level of confidence according to the standard normal distribution, $\mathrm{p}$ = estimated proportion of the population that presents the characteristic, and $\mathrm{e}=$ tolerated margin of error). A convenient sampling technique was followed.

\section{Data collection}

Data was collected using a predesigned online questionnaire distributed to parents/caregivers and included questions designed to fulfill the study objectives.

The questionnaire included questions about the following main items:

- Socio-demographic data of the participants (age, gender, and birth order of the child),

- The personal knowledge of the respondents about nocturnal enuresis and its causes,

- Enuresis-related characteristics like time, relation to sleeping, frequency per week, an improvement in decreasing fluid intake before sleeping, and others,

- Risk factor like a family history of NE in parents or siblings, chronic diseases, anemia, delayed milestones, and others,

- Management modalities used for children who have NE.

\section{Ethical considerations}

The questionnaire has a brief introduction explaining its aim and purpose and informing participants that participation is entirely voluntary. No names were recorded in the surveys, neither date of birth nor address has been collected. All answers were kept confidential and safe.

\section{Statistical analysis}

Data were analyzed using Statistical Package for Social Sciences (SPSS) version 20 (IBM Corp., Armonk, NY). Descriptive statistics were used for the prevalence and quantitative variables. Risk factors were determined using the $\mathrm{X}^{2}$ test ( $\mathrm{x}=$ sample size mean). $\mathrm{P}$-value of less than 0.05 was considered statistically significant.

\section{Results}

The questionnaire was distributed online through social media. Two thousand one hundred and forty-eight (2148) responses where received and analyzed. This study identified a prevalence of enuresis of $31.2 \%$.

Most children (59.6\%) were 5-7 years of age, 58.2\% of the sample were males, and 31.4\% of studied children were the first child (Table 1). The majority (61.3\%) of respondents knew about nocturnal enuresis, and $34.2 \%$ of them believed they know its causes. Nineteen percent identified causes of nocturnal enuresis as weakness 


\section{Cureus}

in the muscles of the lower urinary tract, $9.1 \%$ as problems or damage of the urinary tract or nerves that control the urinary system, $8.0 \%$ as psychological problems, and $2.8 \%$ as urinary tract infection. About a quarter $(25.7 \%)$ of participants had at least one other child suffering from nocturnal enuresis.

\begin{tabular}{|c|c|c|}
\hline & Frequency & Percent \\
\hline \multicolumn{3}{|l|}{ Child age (in years) } \\
\hline 3-5 & 556 & 25.9 \\
\hline $5-7$ & 1280 & 59.6 \\
\hline 7-10 & 216 & 10.1 \\
\hline$>10$ & 94 & 4.4 \\
\hline \multicolumn{3}{|l|}{ Child sex } \\
\hline Female & 898 & 41.8 \\
\hline Male & 1248 & 58.2 \\
\hline \multicolumn{3}{|l|}{ Birth order of the child } \\
\hline 1 & 674 & 31.4 \\
\hline 2 & 416 & 19.4 \\
\hline 3 & 340 & 15.8 \\
\hline $4-5$ & 384 & 17.9 \\
\hline$>5$ & 332 & 15.5 \\
\hline \multicolumn{3}{|l|}{ Participants know about nocturnal enuresis } \\
\hline No & 832 & 38.7 \\
\hline Yes & 1316 & 61.3 \\
\hline \multicolumn{3}{|l|}{ Participants know about its causes } \\
\hline No & 1414 & 65.8 \\
\hline Yes & 734 & 34.2 \\
\hline \multicolumn{3}{|l|}{ Identified causes of nocturnal enuresis (by participants) } \\
\hline Weakness in the muscles of the lower urinary tract & 408 & 18.9 \\
\hline Problems or damage of the urinary tract or nerves that control the urinary system & 196 & 9.1 \\
\hline Psychological problems & 172 & 8.0 \\
\hline Urinary tract infections & 60 & 2.8 \\
\hline Hereditary & 54 & 2.5 \\
\hline Anemia & 12 & .6 \\
\hline Irritability & 18 & .8 \\
\hline Pregnancy and birth-related causes & 8 & .4 \\
\hline \multicolumn{3}{|l|}{ Having a child suffering from nocturnal enuresis } \\
\hline No & 1478 & 68.8 \\
\hline Yes & 670 & 31.2 \\
\hline
\end{tabular}

TABLE 1: Child age, sex, birth order, parents'/caregivers' knowledge about nocturnal enuresis and prevalence of nocturnal enuresis among the studied children 


\section{Cureus}

Table 2 shows nocturnal enuresis-related characteristics among the studied sample. More than $40 \%$ of the children had enuresis at night only, while $55.1 \%$ had it during day and night although $78.8 \%$ of children improved on decreasing fluid intake before sleeping within 5-7 weeks. The problem caused embarrassment and social shame for $94.3 \%$ of studied children, and $76.4 \%$ sought medical advice. Of those $29.6 \%$ received pharmacological treatment, $31.6 \%$ behavioral modification, $6.8 \%$ bedwetting alarm, $6.2 \%$ by exercise, and only $1.5 \%$ had surgery.

\begin{tabular}{|c|c|c|}
\hline Variable & Frequency & Percentage \\
\hline \multicolumn{3}{|l|}{ Time of enuresis } \\
\hline At night only & 294 & 43.9 \\
\hline Day and night & 376 & 55.1 \\
\hline Improvement of decreasing fluids intake before sleeping & 344 & 51.3 \\
\hline \multicolumn{3}{|l|}{ Frequency per week } \\
\hline $1-2$ & 80 & 11.9 \\
\hline 3-4 & 102 & 15.2 \\
\hline $5-7$ & 528 & 78.8 \\
\hline Mother keen to wake the child at night to urinate & 610 & 91.0 \\
\hline The problem causes embarrassment and social shame to the child & 632 & 94.3 \\
\hline Seeking medical advice & 512 & 76.4 \\
\hline \multicolumn{3}{|l|}{ Type of provided treatment } \\
\hline Pharmacological treatment & 198 & 29.6 \\
\hline Surgery & 10 & 1.5 \\
\hline Exercises to strengthen the bladder muscles & 42 & 6.2 \\
\hline Bedwetting alarm & 46 & 6.8 \\
\hline Behavioral modification & 212 & 31.6 \\
\hline Improvement of nocturnal enuresis on different types of treatment & 262 & 43.6 \\
\hline
\end{tabular}

\section{TABLE 2: Nocturnal enuresis-related characteristics among the studied cases}

This study has shown significant relationship between enuresis and child's age $(\mathrm{P}=0.05)$ and gestational age (in months) at birth ( $\mathrm{P}=0.013)$, type of delivery, hospital admission after delivery, sibling suffering from the same condition, birth order of the child, parents' history of NE, diabetes, urinary tract infection, psychological problems and delayed milestones $(\mathrm{P}<0.05)$ (Table 3). While there were no significant correlations between nocturnal enuresis and child gender $(\mathrm{P}=0.104)$.

\begin{tabular}{|c|c|c|c|c|c|}
\hline \multirow{2}{*}{ Variables } & \multirow{2}{*}{ Responses } & \multicolumn{2}{|c|}{ Nocturnal enuresis } & \multirow{2}{*}{ Total $(\mathrm{N}=2148)$} & \multirow{2}{*}{$P$ value } \\
\hline & & Yes $(n=670)$ & No $(n=1478)$ & & \\
\hline \multirow{7}{*}{ Child age (in years) } & & 150 & 408 & 558 & \multirow{7}{*}{0.05} \\
\hline & & $22.4 \%$ & $27.6 \%$ & $26.0 \%$ & \\
\hline & \multirow{2}{*}{$5-7$} & 426 & 854 & 1280 & \\
\hline & & $63.6 \%$ & $57.8 \%$ & $59.6 \%$ & \\
\hline & \multirow{2}{*}{$7-10$} & 64 & 152 & 216 & \\
\hline & & $9.6 \%$ & $10.3 \%$ & $10.1 \%$ & \\
\hline & $>10$ & 30 & 64 & 94 & \\
\hline
\end{tabular}




\section{Cureus}

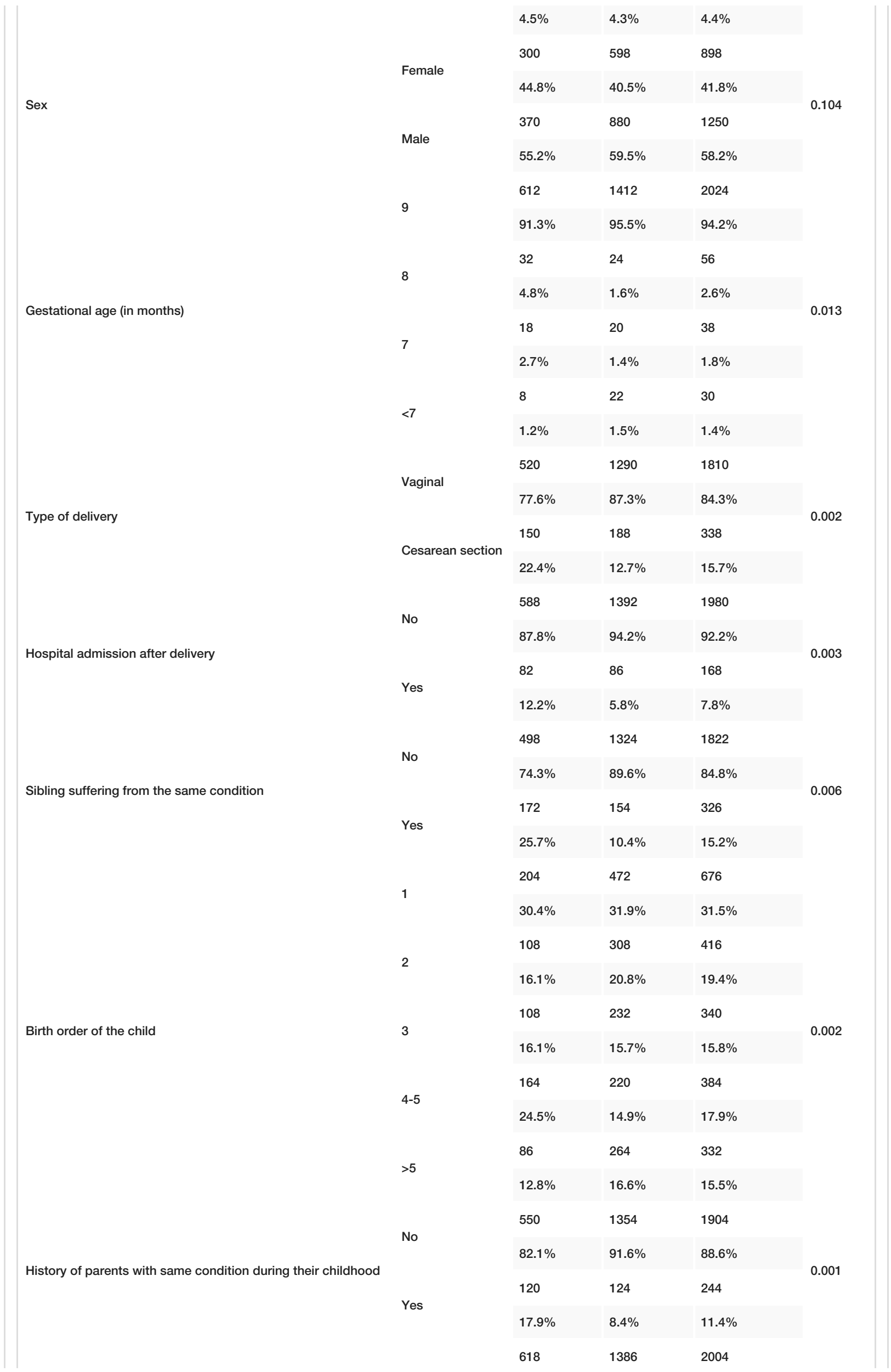




\section{Cureus}

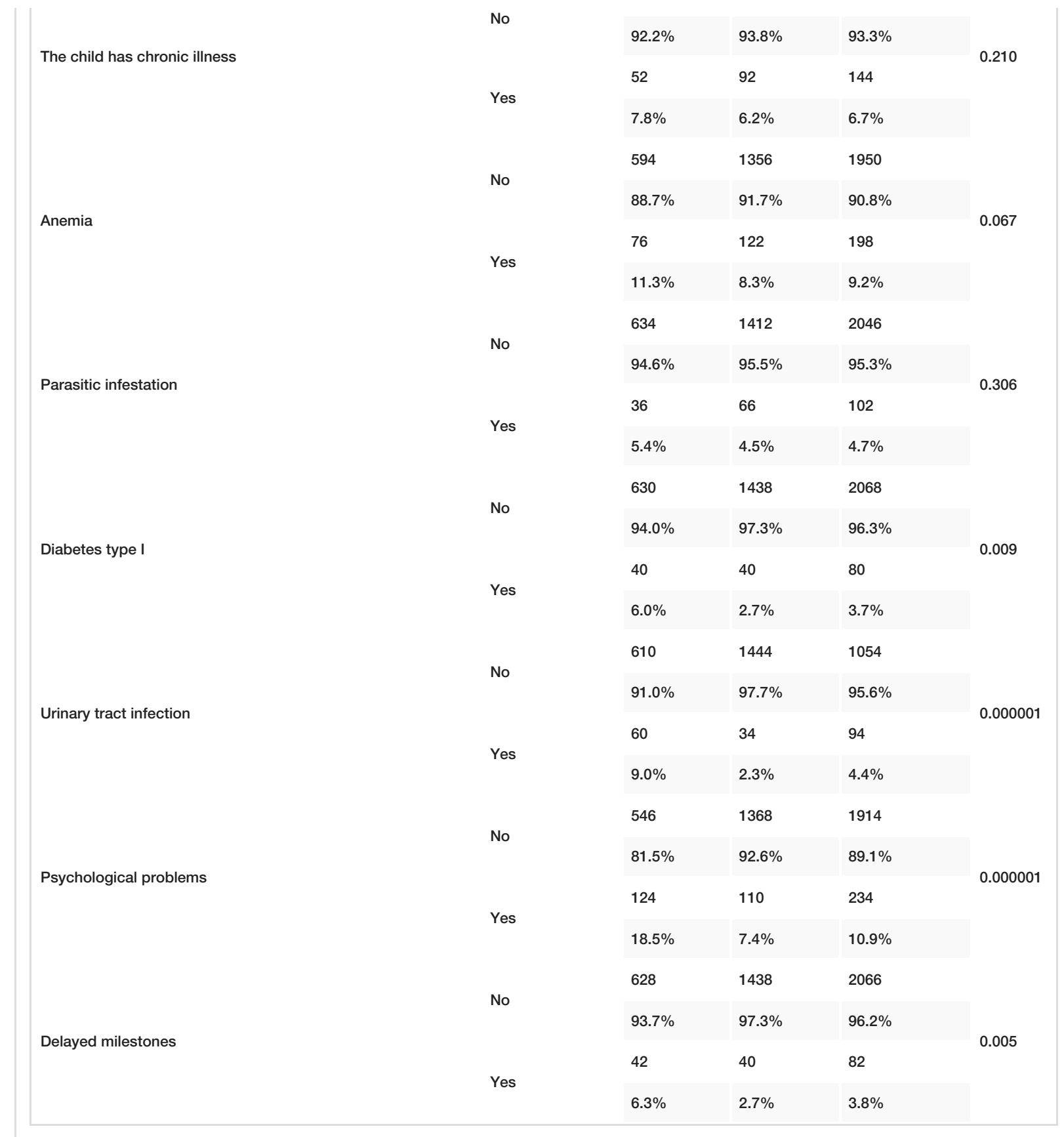

TABLE 3: Risk factors of nocturnal enuresis among the studied children

\section{Discussion}

Nocturnal enuresis (NE) is the involuntary urination that occurs while asleep after an age when bladder control at night is expected. It is more common in children living in unfavorable social conditions who are under psychosocial stress and is known to affect a child's psychological state $[1,10]$. It is commonly identified amongst school-aged children with a significant stressor along with psychosocial problems both for parents and children [1]. Multiple mechanisms have been proposed for NE, including dysfunctional bladder, small functional bladder capacity, abnormal antidiuretic hormone levels, and irregular sleep patterns [11].

This is a cross-sectional study that was conducted among 2148 children in KSA and aimed to show the prevalence, risk factors, provided modalities of treatment for nocturnal enuresis among studied children in KSA.

The prevalence of nocturnal enuresis in children is between 15 and $25 \%$ at five years of age, and it goes down as children grow [3]. In this study, the majority of participants had children aged 5-7 years, of whom 
$63 \%$ had NE, while the prevalence significantly decreased to $9.6 \%$ in children $7-10$ years and $4.5 \%$ in children older than 10 years of age $(P=0.05)$. The prevalence was close to what is reported from Sherah et al. study conducted in Jazan city in Saudi Arabia for children aged 5-12 years which was 76.4\% but was higher than those reported from other studies where the overall prevalence of enuresis was found to be $12.95 \%$ in children aged 5-16 years from France and 15\% in children aged 6-11 years from Saudi Arabia and two studies in Turkey estimated the prevalence to be $12.4 \%$ and $13 \%$ [1,12-15]. However, in India, another survey carried out among 1473 children aged between 6 and 10 years reported that the overall prevalence of enuresis was 7.61\% [16]. In Iran, a systemic review conducted to estimate the prevalence of enuresis and its related factors among Iranian children found that the prevalence of enuresis among all children was estimated as of $11.01 \%$ [17].

Most reported enuresis cases in our study had it at day and night 55.1\%, and 43.9\% occurred at night only. However, Sherah et al. and Sarici et al. reported that daytime enuresis was seen in only $14.29 \%$ and $18 \%$ of cases, respectively, of children of school-age $[12,18]$.

The causation of enuresis is generally multifactorial and is the result of the interaction of physical and psychological factors. Participants believed that enuresis could be caused by weakness in the muscles of the lower urinary tract by $18.9 \%$, problems or damage of the urinary tract or nerves that control the urinary system (9.1\%), psychological problems (8\%), urinary tract infections (2.8\%) and anemia, hereditary, pregnancy, and birth-related causes believed to be caused by small percentage of respondents. Schlomer et al. reported that parents in their study believed the most common cause of enuresis to be deep sleeper (56\%), unknown (39\%), laziness to wake up and go to the bathroom (26\%) and small bladder size (21\%) [19]. This perception did not change a lot from older studies that have mentioned that parents believed heavy sleeping, emotional problems, and small bladder size to be important causes of NE [19,20].

Participants also reported different practices in terms of modalities of treatment provided where behavioral modification was the most commonly used modality by $31.6 \%$, followed by pharmacological treatment (29.6\%), bedwetting alarm (6.8\%), exercises to strengthen the bladder muscles (6.2\%) and surgery reported by $1.5 \%$ only. We found that improvement of nocturnal enuresis on different types of treatment occurred in $43.6 \%$ of cases studied. In contrast to our results, Sherah et al. reported using medical treatment in $76 \%$ of case and Al-Zahrani et al. reported the treatment methods used to be: enuresis alarm, water restriction, medication, and awaking for voiding in $56.9 \%, 14.7 \%, 5.7 \%$ and $5.7 \%$ of cases, respectively [12,20]. Schlomer et al. reported that parents used some behavioral modifications like voiding prior to sleep (77\%), limiting fluid intake at night (71\%), and using bedwetting alarm (6\%) [19].

A cross-sectional survey, performed in Primary Health Care Centers, found that out of 65 families that have children with NE, $38.7 \%$ was the frequency of bedwetting every night, $22.6 \%$ of the children were stressed as a result of new childbirth, $14 \%$ of the families did not feel a family load of having children with NE, $29 \%$ of the families did not try to treat their children because of their improvement with time, and $12 \%$ of the families that tried to treat their children used fluid restriction and waked their children up frequently at night [21].

Regarding risk factors of nocturnal enuresis among the studied children, our study found significant correlations between nocturnal enuresis and child age as the prevalence was significantly lower as children grew older (peak is 63.6\% in 5-7 years old), and no significant relation was found between enuresis and gender $(p=0.104)$, which was also reported by a study done in Taif that has shown a prevalence of $7.33 \%$ and $8.42 \%$ in boys and girls, respectively [20]. In contrast to Bakhtiar et al. who reported the prevalence of nocturnal enuresis in boys $(10.7 \%)$ to be higher than in girls $(5.4 \%)(P=0.009)$ [22]. However, there was a significant correlation with a parent suffering from the same condition in their childhood $(\mathrm{p}=0.001)$. Another study reported that the prevalence of enuresis among boys was 1.65-fold greater than that of girls, and it was more common among children with positive familial history [17]. Another study found that the prevalence of enuresis was found to correlate well with age $(p=0.0001)$, but not correlate with gender $(p>$ $0.05)$ [1]. In addition, Bakhtiar et al. reported statistically significant relationships between nocturnal enuresis and history of nocturnal enuresis in siblings $(\mathrm{P}=0.023)$ and deep sleep $(\mathrm{P}=0.007)$ amongst other factors [22].

\section{Conclusions}

Our study reported that $31.2 \%$ of children suffer from nocturnal enuresis, but there were no significant correlations between nocturnal enuresis and child gender. There was a significant correlation with child age and family history of NE in parents or siblings. Behavioral modification therapy was the most provided treatment followed by pharmacological treatment and the improvement occurred in less than half of the cases. So, we recommend health education about the causes and risk factors in addition to encouraging prompt treatment and close follow-up to prevent associated self-shame and family stress. Further studies are needed to look in-depth into details of the modalities of treatment and how they are conducted and followed in addition to their effectiveness in Saudi children. Families' compliance with those modalities in Saudi culture is worth further investigation. 


\section{Additional Information \\ Disclosures}

Human subjects: Consent was obtained by all participants in this study. IRB of Princess Nourah Bint Abdulrahman University KACST, KSA: H-01-R-059 issued approval 19-0145. Animal subjects: All authors have confirmed that this study did not involve animal subjects or tissue. Conflicts of interest: In compliance with the ICMJE uniform disclosure form, all authors declare the following: Payment/services info: All authors have declared that no financial support was received from any organization for the submitted work. Financial relationships: All authors have declared that they have no financial relationships at present or within the previous three years with any organizations that might have an interest in the submitted work. Other relationships: All authors have declared that there are no other relationships or activities that could appear to have influenced the submitted work.

\section{References}

1. Gür E, Turhan P, Can G, et al.: Enuresis: prevalence, risk factors and urinary pathology among school children in Istanbul, Turkey. Pediatr Int. 2004, 46:58-63. 10.1111/j.1442-200X.2004.01824.x

2. Özkan S, Durukan E, Iseri E, Gürocak S, Maral I, Bumin MA: Prevalence and risk factors of monosymptomatic nocturnal enuresis in Turkish children. Indian J Urol. 2010, 26:200-205. 10.4103\%2F0970-1591.65387

3. von Gontard A, Mauer-Mucke K, Plück J, Berner W, Lehmkuhl G: Clinical behavioral problems in day- and night-wetting children. Pediatr Nephrol. 1999, 13:662-667. 10.1007/s004670050677

4. Hamed A, Yousf F, Hussein MM: Prevalence of nocturnal enuresis and related risk factors in school-age children in Egypt: an epidemiological study. World J Urol. 2017, 35:459-465. 10.1007/s00345-016-1879-2

5. Ghotbi N, Kheirabadi GH: Prevalence of nocturia and its associated factors in primary school children in Sanandaj in 2002. J Kurdistan Univ Med Sci. 2001, 50:30-33.

6. Yousef K, Basaleem H, Yahiya M: Epidemiology of nocturnal enuresis in basic schoolchildren in Aden Governorate, Yemen. Saudi J Kidney Dis Transpl. 2011, 22:167-173.

7. Jamali M, Rafiei GH: A comparison between the results of treatment with imipramine and oxybutynin in non-symptomatic 6-12 children with enuresis. J Rafsanjan Univ Med Sci Health Serv. 2004, 3:113-118.

8. Theunis M, Van Hoecke E, Paesbrugge S, Hoebeke P, Vande Walle J: Self-image and performance in children with nocturnal enuresis. Eur Urol. 2002, 41:660-667. 10.1016/S0302-2838(02)00127-6

9. Elgohary H, Shalanda A, El lethy S: Prevalence and psychiatric comorbidities of nocturnal enuresis in a sample of basic education students in Sharkia governorate. Zagazig Univ Med J. 2013, 19:1-9. 10.21608/zumj.2013.4291

10. Melville JL, Walker E, Katon W, Lentz G, Miller J, Fenner D: Prevalence of comorbid psychiatric illness and its impact on symptom perception, quality of life, and functional status in women with urinary incontinence. Am J Obstetric Gynecol. 2002, 187:80-87. 10.1067/mob.2002.124839

11. Hjalmas K: Nocturnal enuresis: basic facts and new horizons . Eur Urol. 1998, 33:53-57.

12. Sherah K, Elsharief M, Barkat N, Jafery A: Prevalence of nocturnal enuresis in school-age children in Saudi Arabia. Int J Med Develop Ctries. 2019, 3:669-675. 10.24911/IJMDC.51-1547073432

13. Ouedraogo A, Kere M, Ouedraogo TL, Jesu F: Epidemiology of enuresis in children and adolescents aged 516 years in Ouagadougou (Burkina Faso) (Article in French). Arch Pediatr. 1997, 4:947-951. 10.1016/S0929693X(97)86089-7

14. Kalo BB, Bella H: Enuresis: prevalence and associated factors among primary school children in Saudi Arabia. Acta Pediatr. 1996, 85:1217-1222. 10.1111/j.1651-2227.1996.tb18232.x

15. Gumus B, Vurgun N, Lekili M, Işcan A, Muezzinoglu T, Buyuksu C: Prevalence of nocturnal enuresis and accompanying factors in children aged 7-11 years in Turkey. Acta Paediatr. 1999, 88:1369-1372. 10.1080/080352599750030103

16. De Sousa A, Kapoor H, Jagtap J, Sen M: Prevalence and factors affecting enuresis amongst primary school children. Indian J Urol. 2007, 23:354-357. 10.4103/0970-1591.36703

17. Makrani A, Moosazadeh M, Nasehi M, et al.: Prevalence of enuresis and its related factors among children in Iran: a systematic review and meta-analysis. Int J Pediatr. 2015, 3:995-1004. 10.22038/ijp.2015.5142

18. Sarici H, Telli O, Ozgur B, Demirbas A, Ozgur S, Karagoz MA: Prevalence of nocturnal enuresis and its influence on quality of life in school-aged children. J Pediatr Urol. 2016, 12:159.e1-159.e6. 10.1016/j.jpurol.2015.11.011

19. Schlomer B, Rodriguez E, Weiss D, Copp H: Parental beliefs about nocturnal enuresis causes, treatments, and the need to seek professional medical care. J Pediatr Urol. 2013, 9:1043-1048. 10.1016/j.jpurol.2013.02.013

20. Al-Zahrani SS: Nocturnal enuresis and its treatment among primary-school children in Taif, KSA . Int J Res Med Sci. 2014, 2:91-94. 10.5455/2320-6012.ijrms20140218

21. Alshahrani A, Selim M, Abbas M: Prevalence of nocturnal enuresis among children in Primary Health Care Centers of Family and Community Medicine, PSMMC, Riyadh City, KSA. J Family Med Prim Care. 2018, 7:937-941.

22. Bakhtiar K, Pournia Y, Ebrahimzadeh F, Farhadi A, Shafizadeh F, Hosseinabadi R: Prevalence of nocturnal enuresis and its associated factors in primary school and preschool children of Khorramabad in 2013. Int J Pediatr. 2014, 2014:7. 10.1155/2014/120686 